\title{
Editorial: COVID-19: Emergence, Spread, and Implication for Public Health
}

\author{
Ali Alami ${ }^{* *}$ (1) \\ 1. Department of Epidemiology and Biostatistics, School of Public Health, Social Determinants of Health Research Center, Gonabad University of \\ Medical Sciences, Gonabad, Iran.
}

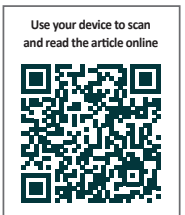

Citation Ali Alami. COVID-19: Emergence, Spread, and Implication for Public Health. Journal of Research \& Health. 2020; 10(3):131-134. http://dx.doi.org/10.32598/JRH.10.3.303.4

doij http://dx.doi.org/10.32598/JRH.10.3.303.4

uring the $21^{\text {st }}$ century, human beings have encountered three major diseases caused by newly discovered coronaviruses called Severe Acute Respiratory Syndrome (SARS), the Middle East Respiratory Syndrome (MERS), and coronavirus disease-2019 (COVID-19).

COVID-19, declared a pandemic by the World Health Organization [1], emerged in Wuhan, China in the last days of 2019 and rapidly spread around the world. There are more than 4400000 cases of COVID-19 and 298000 deaths attributed to the disease until May 14, 2020. In Iran, nearly 113000 cases and more than 6700 deaths have been reported to this date [2].

Despite the similarities between the three diseases in terms of the causative agent, the modes of transmission, and the main symptoms, the impact of COVID-19 on public health is very different from SARS and MERS for several reasons. The rapid global spread of COVID-19, the high fatality rate, and the different nature of the virus in terms of persistence in the environment could be considered as the reasons for this difference.

To control the disease, various precautions have recommended such as hand washing, social distancing, social isolation, use of personal protective equipment, school/university closure, working at home, cocooning the vulnerable groups such as the elderly, and travel ban [3]. But, these pieces of advice could also have some negative impacts on human life. The social, economic, and cultural consequences of these recommendations could also endanger human public health in the short and long term $[4,5]$. Moreover, the main health implications due to implementation of the COVID-19 recommendation are psychological impacts such as psychological distress [6], anxiety [7, 8], depression [9], and insomnia [10] among people, students, and medical staff. As providing qualified care for health service recipients is important, it seems that evaluation and control of the psychological impact among health care professionals have a special priority.

There may also be negative public health effects because of the persistence of COVID-19. Indeed, people may fear of potential transmission of the virus while visiting the hospitals, health centers, and health units, even when receiving the required services $[11,12]$. Therefore, the necessary services are not received on time, and this could lead to complications in the public health of the community.

One of the most important health care services is immunization services [13]. Lack of adequate attention

* Corresponding Author:

Ali Alami, PhD.

Address: Department of Epidemiology and Biostatistics, School of Public Health, Social Determinants of Health Research Center, Gonabad University of Medical Sciences, Gonabad, Iran.

Phone: +98(915) 1779214

E-mail: alami.ali@gmu.ac.ir 
to the immunization program of less than 24-month old children, especially in poor countries and the poor population of rich countries could lead to the loss of all positive vaccination achievements over the past decades. Inadequate attention to immunization can even lead to the return of epidemic-prone diseases such as measles, rubella, polio, diphtheria, and pertussis. WHO asserts that "if immunization services are compromised due to severe limitation of health-care resources, vaccines for these diseases may need to be prioritized" [14]. Although the sustenance of routine vaccination of the target groups would be vital to maintain public health, health services have an important responsibility to protect all health workers $[14,15]$. So, while emphasizing the continuity of the immunization program, providing suitable workspace for immunization with the least risk of contamination to the vaccine recipients, as well as providing effective personal protective equipment for the vaccinators would be essential.

Another important group that may be affected by the COVID-19 pandemic is the elderly [16]. Social isolation may protect the elderly from getting COVID-19, but there are serious public health concerns. Indeed, the risk of their cardiovascular, mental health, autoimmune, and neurocognitive problems may increase during cocooning [17]. They may also be deprived of receiving routine services due to isolation, especially those who do not have close family and or friends. To prevent these problems, the health system may need to run an active health care program through health centers or to launch and enhance a voluntary supporting service for the elderly [16].

In general, the COVID-19 pandemic has affected all aspects of human life, including public health. We do not know when the COVID-19 pandemic will end. So, proper intervention planning and adequate attention to medical staff as well as vulnerable groups in society, including children and the elderly, are needed to reduce the implications of the pandemic. In this regard, the role of international health agencies and the responsibility of governments as well as researchers to provide credible research evidence for informed decisions would be very vital and significant.

\section{Ethical Considerations}

\section{Compliance with ethical guidelines}

This is a letter to editor and there was no ethical considerations to be considered in this paper.

\section{References}

[1] WHO. Director-General's opening remarks at the media briefing on COVID-19 - 11 March 2020. https://www.who. int/dg/speeches/detail/who-director-general-s-openingremarks-at-the-media-briefing-on-covid-19---11-march-2020. Accessed: 5/15/2020.

[2] worldometers. Accessed: Last updated: June 28, 2020. https://www.worldometers.info/coronavirus/ .

[3] WHO. Coronavirus disease (COVID-19) advice for the public. Accessed: 5/14/2020. https://www.who.int/emergencies/ diseases/novel-coronavirus-2019/advice-for-public.

[4] Asim M, Sathian B, van Teijlingen E, Mekkodathil A, Subramanya SH, Simkhada P. COVID-19 Pandemic: Public Health Implications in Nepal. Nepal J Epidemiol. 2020; 10(1):817-20. [DOI:10.3126/nje.v10i1.28269] [PMID] [PMCID]

[5] Gallego V, Nishiura H, Sah R, Rodriguez-Morales AJ. The COVID-19 outbreak and implications for the Tokyo 2020 Summer Olympic Games. Travel Med Infect Dis. 2020; 34:101604. [DOI:10.1016/j.tmaid.2020.101604] [PMID] [PMCID]

[6] Qiu J, Shen B, Zhao M, et al. A nationwide survey of psychological distress among Chinese people in the COVID-19 epidemic: Implications and policy recommendations. Gen Psychiatry. 2020; 33:e100213. [DOI:10.1136/gpsych-2020-100213] [PMID] [PMCID]

[7] Wenjun Cao, Ziwei Fang, Guoqiang Hou, Mei Han, Xinrong $\mathrm{Xu}$, Jiaxin Dong, Jianzhong Zheng. The psychological impact of the COVID-19 epidemic on college students in China. Psychiatry Res. 2020; 287:112934. [DOI:10.1016/j.psyy chres.2020.112934.] [PMID] [PMCID]

[8] Shanafelt T, Ripp J, Trockel M. Understanding and addressing sources of anxiety among health care professionals during the COVID-19 pandemic. JAMA. 2020. Published online. [DOI:10.1001/jama.2020.5893] [PMID]

[9] Wang C, Pan R, Wan X, Tan Y, Xu L, Ho C.S, Ho R.C. Immediate psychological responses and associated factors during the initial stage of the 2019 Coronavirus Disease (COVID-19) epidemic among the general population in China. Int J Environ Res Public Health. 2020; 17(5):1729. [DOI:10.3390/ijerr ph17051729] [PMID] [PMCID]

[10] WHO. Substantial investment needed to avert mental health crisis. Accessed: 5/14/2020. https://www.who.int/ news-room/detail/14-05-2020-substantial-investment-needed-to-avert-mental-health-crisis.

[11] Molica M, Mazzone C, Cordone I, Pasquale A, Niscola P, Fabritiis P. SARS-COV-2 infection anxieties and general population restrictions delay diagnosis and treatment of acute hematological malignancies. Br J Haematol. 2020; 10.1111/ bjh.16785. [DOI:10.1111/bjh.16785] [PMID] [PMCID]

[12] Lazzerini M, Barbi E, Apicella A, Marchetti F, Cardinale F, Trobia G. Delayed access or provision of care in Italy resulting from fear of COVID-19. The Lancet. 2020; 4:10-11. [DOI:10.1016/S2352-4642(20)30108-5]

[13] WHO. Guidance on routine immunization services during COVID-19 pandemic in the WHO European Region, 20 March 2020. Accessed: 5/14/2020. http://www.euro.who. int/en/health-topics/communicable-diseases/rotavirus/ publications/2020/guidance-on-routine-immunization- 
services-during-covid-19-pandemic-in-the-who-europeanregion,-20-march-2020

[14] WHO. Routine immunization service during the COVID-19 pandemic. Accessed: 5/14/2020. https://apps.who. int/iris/handle/10665/331925

[15] Editorial. COVID-19: protecting health-care workers. Lancet. 2020 21-27 March; 395(10228):922. [DOI:10.1016/S01406736(20)30644-9]

[16] Armitage R, Nellums L.B. COVID-19 and the consequences of isolating the elderly. The Lancet. 2020; 5. [DOI:10.1016/ S2468-2667(20)30061-X.]

[17] Gerst-Emerson K, Jayawardhana J. Loneliness as a public health issue: The impact of loneliness on health care utilization among older adults. Am J Public Health. 2015; 105:10139. [DOI:10.2105/AJPH.2014.302427] [PMID] [PMCID] 
This Page Intentionally Left Blank 